\title{
Extrapolation of proton-proton cross section to cosmic ray energies using geometrical model
}

\author{
Zbigniew Plebaniak ${ }^{1, \mathrm{a}}$ and Tadeusz Wibig ${ }^{1,2, \mathrm{~b}}$ \\ 1 National Centre for Nuclear Research, Astrophysics Division, Cosmic Ray Laboratory, ul. 28 Pułku Strzelców Kaniowskich 69, \\ 90-558 Łódź, Poland \\ 2 Faculty of Physics and Applied Informatics, University of Łódź, ul. Pomorska 149/153, 90-236 Łódź, Poland
}

\begin{abstract}
We present a new parameterization of the hadronic matter distribution in the geometrical picture of elastic scattering. Our model contains four free energy-dependent parameters that allows us to propose a better description of differential elastic cross section data measured in accelerator experiments from ISR to LHC energies. A simple linear dependence of all parameters on the logarithm of energy above $\sqrt{s}=300 \mathrm{GeV}$ allows an extrapolation of elastic, inelastic and total proton-proton cross sections to ultra high energies seen in cosmic rays. Results of the extrapolation are in agreement with cross sections presented by ground-based cosmic ray experiments like AUGER or Telescope Array. Our parameterization also shows agreement with "BEL behaviour" of proton.
\end{abstract}

\section{Introduction}

The hadronic cross section is one of the most important parameter of models dedicated to describing Extensive Air Shower (EAS) development at ultra high energies. It is necessary for example to distinguish between EAS produced by lighter and heavier nuclei. It is also strongly connected with the multiplicity of produced particles. Unfortunately, due to QCD nature it is not possible to calculate this parameter from fundamental physical laws. This fact forces building complicated phenomenological models, based on accelerator measurements at lower energies, that allow for cross section extrapolations to energies observed in cosmic rays. Such models can also give information about the internal structure of colliding particles.

In this paper we concentrate on the problem of extrapolating hadronic cross section to cosmic ray energies. For this purpose we developed optical geometrical picture, assume energy dependent parameterization of the hadronic matter distribution in protons. The presented 4-parameter model describes the distribution of two hadronic matter fractions in protons. We received the energy dependence of parameters based on fits to the differential elastic cross section from accelerator data.

Elastic scattering of hadrons has been studied experimentally in a wide energy range. This work is based on data taken by ISR, SPS, Tevatron and LHC experiments. The energy of $\sqrt{s}=8 \mathrm{TeV}$ for proton-proton collistions at TOTEM and ATLAS experiments is today the highest available energy useful for our considerations. Building our model we obtain the simple logarythmic dependence of four parameteres for higher energies, that allows for extrapolation to cosmic ray energies.

\footnotetext{
a e-mail: zp@zpk.u.lodz.pl

b e-mail: t.wibig@gmail.com
}

The material is organised as follows. In Sect. 2 we present the phenomenological description of the scattering process and eikonal representation of the elastic scattering amplitude. In Sect. 3 we describe the model assumptions. Section 4 is devoted to the results of fits and extrapolation of the cross section. Discussion and physical interpretation are the content of Sect. 5 .

\section{Scattering amplitudes and their eikonal representation}

The differential elastic cross section is described by the elastic scattering amplitude $F(s, t)$ as follows:

$$
\frac{d \sigma_{e l}}{d|t|}=\pi|F(t)|^{2}
$$

We want to depict the absorption processes in a geometrical framework. The relation between interaction geometry and the momentum transfer space is defined with the Fourier transform with the help of the profile function $\Gamma(s, b)[1]$.

$$
\begin{aligned}
F(s, t) & =i \int_{0}^{\infty} J_{0}(b \sqrt{-t}) \Gamma(s, b) b d b \\
& =i \int_{0}^{\infty} J_{0}(b \sqrt{-t})\{1-\exp [-\chi(s, b)]\} b d b
\end{aligned}
$$

where $q^{2}=-t$ is the four-momentum transfer, $b$ - the impact parameter.

The Eikonal $\chi(s, b)$ may be expressed by:

$$
\chi(s, b)=(\lambda(s)+i) \Omega(b, s)
$$


The complex form of this function is defined by the energy-dependent parameter $\lambda(s)$ which represents the ratio of the real to the imaginary part of $\Omega(s, b)$ :

$$
\lambda(s)=\frac{\Re(\Omega(b, s))}{\Im(\Omega(b, s))} .
$$

In our calculations, we used a slightly modified energy dependence of $\lambda$ parameterized, e.g., by Menon in Refs. [2,3]. In Fig. 2 we present, related to $\lambda$, the calculated ratio of the real and the imaginary part of the scattering amplitude $\rho=\Re(F(\mathbf{b}=0, s)) / \mathfrak{I}(F(\mathbf{b}=0, s))$, for central collisions (two dimensional impact parameter $\mathbf{b}=0$ ), with accelerator data.

Using this formalism, cross sections can be described as follows [4]:

$$
\begin{array}{r}
\sigma_{\text {tot }}(s)=2 \int\left[1-\Re\left(e^{i \chi(b, s)}\right)\right] d^{2} \mathbf{b} \\
\sigma_{e l}(s)=\int\left[1-e^{i \chi(b, s)}\right]^{2} d^{2} \mathbf{b} \\
\sigma_{\text {inel }}(s)=\int 1-\left[e^{i \chi(b, s)}\right]^{2} d^{2} \mathbf{b}
\end{array}
$$

The transmission coefficient $\Omega(b, s)$ which means the opaqueness of two colliding hadrons in impact parameter space, was introduced by Chou and Yang in 1968 [5], and can be described by the following convolution:

$$
\Omega(b)=\Omega(\mathbf{b})=i K_{p p} \int_{-\infty}^{\infty} \int_{0} D\left(\mathbf{b}-\mathbf{b}^{\prime}\right) D\left(\mathbf{b}^{\prime}\right) d^{2} \mathbf{b}^{\prime},
$$

where $D(b)$ is a hadron profile function representing the integral of the density function $\rho(x, y, z)$ in the $z$ direction as a collision axis:

$$
D(\mathbf{b})=\int_{-\infty}^{\infty} \rho(x, y, z) d z
$$

One of the simplest hadronic matter distributions $\rho(x, y, z)$ we can imagine is the one used in [6]. It represents the exponential decreasing of hadron matter density with radius:

$$
\rho_{h}(\mathbf{r})=\frac{m_{h}^{3}}{8 \pi} e^{-m_{h}|\mathbf{r}|}
$$

Results of the scattering cross section obtained using this form shows agreement with accelerator data below and at the region of the first dip. Above this region, the deficit of higher transfer momenta $\left(|t|>1 \mathrm{GeV}^{2}\right)$ can be observed. Currently, in the light of new LHC data at $\sqrt{s}$ of 7 and $8 \mathrm{TeV}$ we tried to update the model modifying the distribution described by Eq. (10).

\section{Model}

To obtain a more satisfying description of the elastic scattering, we introduced the sum of two exponents with different slopes, $m_{1}$ and $m_{2}$, and two differrent normalization factors, $c_{1}$ and $c_{2}$, instead of one in Eq. (10).

Using Eq. (11), experimental data between $20 \mathrm{GeV}$ and $8 \mathrm{TeV}$ were fitted. Four parameters $m_{1,2}$ and $c_{1,2}$
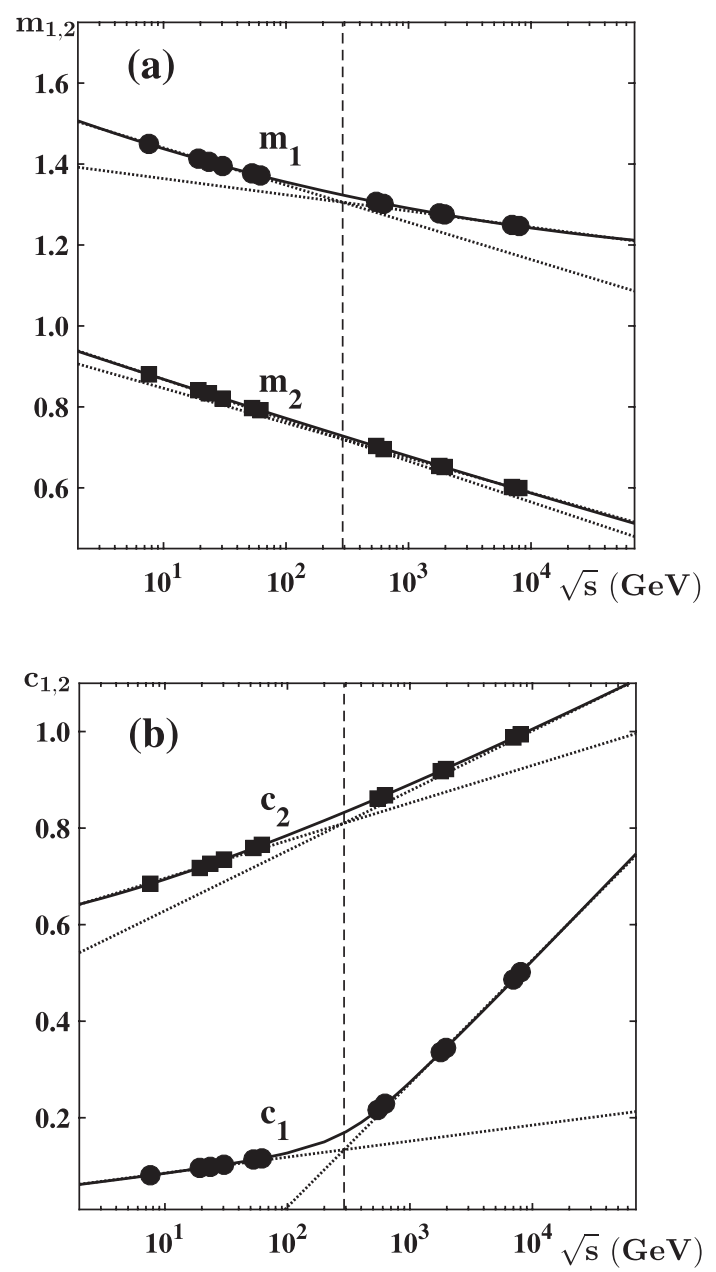

Figure 1. The values of adjusted parameters $m_{1,2}$ (a) and $c_{1,2}$ (b) are shown by the solid symbols. Dashed lines show the asymptotic linear energy dependencies (for high and low energies) of all parameters. Solid lines are the smooth overall energy dependencies.

were found for ten energy data sets starting from $\sqrt{s}=20 \mathrm{GeV}$, through five energies of ISR, SPS point at $546 \mathrm{GeV}$, Tevatron at 0.6 and $2 \mathrm{TeV}$ and LHC data set measured at 7 and $8 \mathrm{TeV}$ in centre of mass [7]. Results are shown in Fig. 1.

$$
\rho_{h}(\mathbf{r})=\frac{1}{8 \pi}\left(c_{1} m_{1}^{3} e^{-m_{1}|\mathbf{r}|}+c_{2} m_{2}^{3} e^{-m_{2}|\mathbf{r}|}\right) .
$$

The integral of this distribution may be expressed by analytic function as:

$$
D(r(x, y))=c_{1} \frac{m_{1}^{2}}{4 \pi} m_{1} r K_{1}\left(m_{1} r\right)+c_{2} \frac{m_{2}^{2}}{4 \pi} m_{2} r K_{1}\left(m_{2} r\right)
$$

The first fraction, with higher slope $m_{1}$, represents the hard inner core of proton. Its opacity related to normalisation $c_{1}$ is going up much faster with energy over $\sqrt{s}=300 \mathrm{GeV}$. The second fraction, $m_{2}, c_{2}$, represents a softer and bigger hadron matter cloud. All four parameters indicate a change around $300 \mathrm{GeV}$ in the center of mass energy which indicates that some new processes start at this point. The decrease of both slopes and the increase of both normalisation factors when the collision energy rise, confirm that the proton becoms Blacker, Edgier and Larger according to postulated BEL behaviour. 
Table 1. The extrapolated cross sections in $\mathrm{mb}$ at higher energies.

\begin{tabular}{|c|c|c|c|c|c|c|}
\hline Energy $(\sqrt{s})$ & $8 \mathrm{TeV}$ & $14 \mathrm{TeV}$ & $24 \mathrm{TeV}$ & $30 \mathrm{TeV}$ & $57 \mathrm{TeV}$ & $95 \mathrm{TeV}$ \\
\hline Fagundes et al. [29] & & $108.6 \pm 1.2$ & & & & \\
\hline Bourelly et al. [30] & & $103.63 \pm 1.0$ & & & & \\
\hline Petrov et al. [31] & & 106.73 & & & & \\
\hline Block, Halzen et al. [32] & & 107.30 & & & & \\
\hline Islam et al. [33] & & 110.00 & & & & \\
\hline Jenkovszky et al. [34] & & 111.00 & & & & \\
\hline Block [35] & & & & & $133.40 \pm 1.6$ & \\
\hline AKENO [28] & $101 \pm 16$ & $104 \pm 26$ & $124 \pm 34$ & & & \\
\hline Fly's Eye [26] & & & & $120 \pm 15$ & & \\
\hline AUGER [18] & & & & & $133.20 \pm 13$ & \\
\hline Telescope Array [22] & & & & & & $170.00 \pm 50$ \\
\hline LHC TOTEM [36] & 102.9 & & & & & \\
\hline LHC ATLAS [37] & 96.07 & & & & & \\
\hline presented model & 96.95 & 105.56 & 115.8 & 120.33 & 132.66 & 143.09 \\
\hline
\end{tabular}

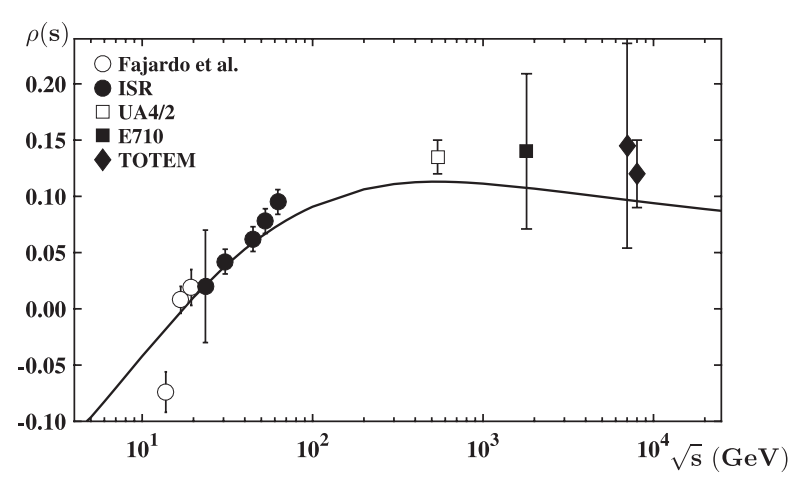

Figure 2. Ratio of the real and the imaginary part of the scattering amplitude $\rho=\Re(F(\mathbf{b}=0, s)) / \Im(F(\mathbf{b}=0, s))$. The solid curve is the result of the parameterisation of $\lambda(s)$ from Ref. [2,3] modified slightly by us used in the present work. Points represent data from [8-12].

\section{Results}

Using the presented model, we calculated the elastic, inelastic and total cross sections according to Eqs. (6), (7) and (5). Their compatibility with experimental data is presented in Fig. 5.

In Fig. 3 we plotted the calculated elastic scattering amplitudes related to the measured differential elastic cross sections at three energies. We can see an agreement with data over the first dip region which was a weakness of models prepared in past.

We used the calculated amplitudes such as in Fig. 3 to determine low $|t|$ slopes. In Fig. 4 results of our model are presented in good agreement with published data. Points are fits to the experimental results in the range of $0.1<$ $|t|<0.3 \mathrm{GeV}^{2}$.

\section{Discussion}

We have developed a modified simple optical model of protonproton scattering with four model parameters, which allows us to describe the hadronic matter distribution of colliding protons. Values of all the parameters were adjusted to the elastic scattering data in the wide range of energies from fixed target experiments below the ISR to the $8 \mathrm{TeV}$ energy of LHC $|t|$ distribution data. Using the eikonal parameterization the correct description of the total and elastic cross sections, the elastic slope and

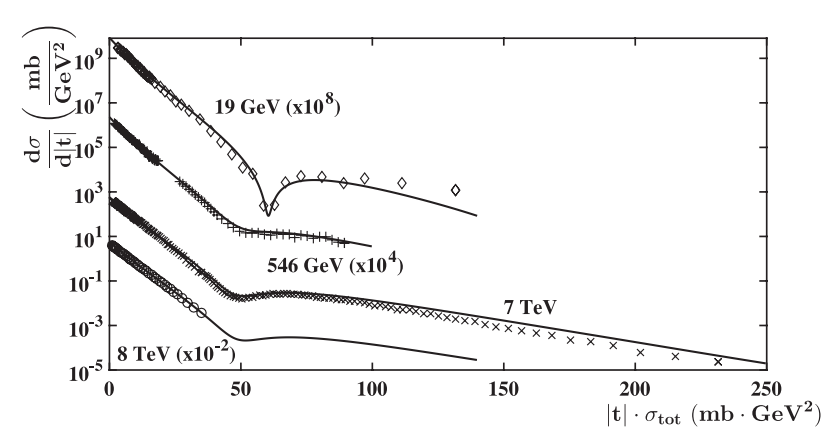

Figure 3. The differential elastic cross sections from our model for c.m.s energies of $19 \mathrm{GeV}, 546 \mathrm{GeV}$ and $7 \mathrm{TeV}$ shown as a function of the $\left(|t| \times \sigma_{\text {tot }}\right)$ compared with the measurements [13-17].

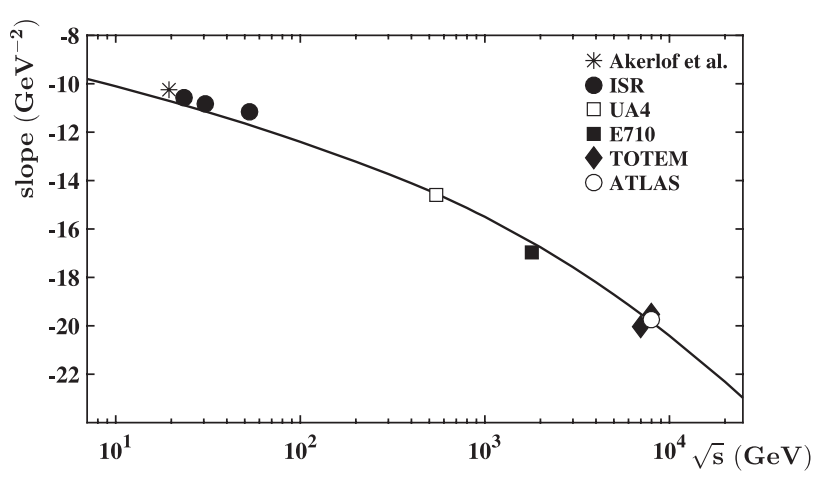

Figure 4. Calculated slopes of the elastic differential cross section as a function of interaction energy. Line shows our model predictions, points are fits to the experimental results from $[13,16-21]$ in the range of $0.1<|t|<0.3 \mathrm{GeV}^{2}$.

the differential elastic cross section at large values of momentum transfer have been found.

We found that for the low and the high energies there are asymptotic regimes which seem to be linear in the logarithmic scale of the available interaction energy $\sqrt{s}$. In agreement with "BEL" behaviour our model shows that the proton becomes blacker and larger as the energy increases.Thanks to the new parameterization of the hadronic matter distribution we obtain better agreement with scattering differential cross section accelerator data for higher momenta transfers for all available energies. 


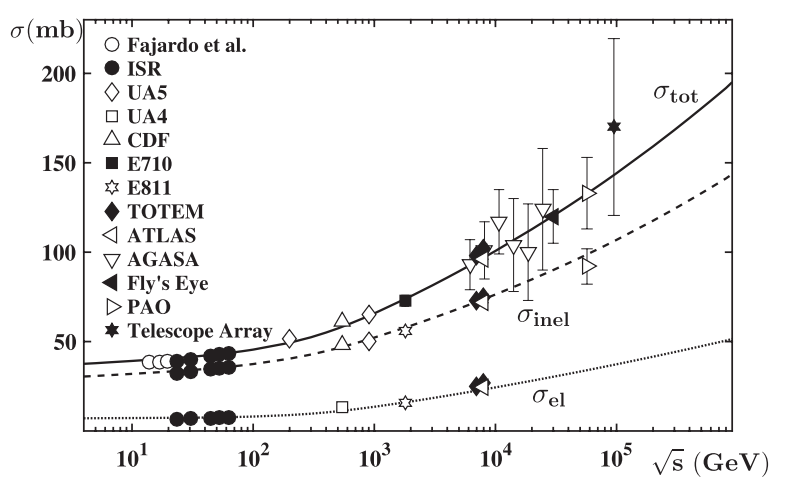

Figure 5. Values of the elastic, inelastic and total cross section calculated with our model as a function of the interaction energy compared with the measurements. The solid line represents total cross section, dashed inelastic and dotted line elastic cross section predictions. Points are experimental results from Refs. [8-10,12, $18,20,22-28]$.

It constitutes a solid base for the extrapolation of the above scattering cross sections available from accelerator measurements at present, up to very high energy domains of cosmic rays and could help us to interpret the data from an astrophysical and a high energy physics point of view.

\section{References}

[1] V. Barone, E. Predazzi High-energy particle diffraction (Berlin: Springer, 2002), ISBN 3-540-42107-6

[2] D. Fagundes, M. Menon, G. Silva, The European Physical Journal C 71, 1637 (2011)

[3] A.F. Martini, M.J. Menon, Phys. Rev. D 56, 4338 (1997)

[4] T. Wibig, D. Sobczynska, Journal of Physics G: Nuclear and Particle Physics 24, 2037 (1998)

[5] T.T. Chou, C.N. Yang, Phys. Rev. 170, 1591 (1968)

[6] T. Wibig, Journal of Physics G: Nuclear and Particle Physics 39, 085003 (2012)

[7] Z. Plebaniak, T. Wibig, Physics Letters B 761, 469 (2016)

[8] U. Amaldi, K. Schubert, Nuclear Physics B 166, 301 (1980)

[9] N.A. Amos et al. (E710 Collaboration), Phys. Rev. Lett. 68, 2433 (1992)

[10] G. Antchev et al. (TOTEM Collaboration), Europhys. Lett. 101, 21004 (2013)

[11] C. Augier et al. (UA4/2 Collaboration), Physics Letters B 316, 448 (1993)

[12] L.A. Fajardo, R. Majka, J.N. Marx, P. Némethy, L. Rosselet, J. Sandweiss, A. Schiz, A.J. Slaughter, C. Ankenbrandt, M. Atac et al., Phys. Rev. D 24, 46 (1981)
[13] C.W. Akerlof et al., Phys. Rev. D14, 2864 (1976)

[14] G. Antchev et al. (TOTEM Collaboration), EPL (Europhysics Letters) 95, 41001 (2011)

[15] G. Antchev et al. (TOTEM Collaboration), EPL (Europhysics Letters) 101, 21002 (2013)

[16] R. Battiston et al. (UA4 Collaboration), Phys. Lett. B127, 472 (1983), [141(1983)]

[17] M. Bozzo et al. (UA4 Collaboration), Phys. Lett. B155, 197 (1985)

[18] P. Abreu et al. (The Pierre Auger Collaboration), Phys. Rev. Lett. 109, 062002 (2012)

[19] N.A. Amos et al. (E-710 Collaboration), Phys. Lett. B247, 127 (1990)

[20] G. Antchev et al. (TOTEM Collaboration), Phys. Rev. Lett. 111, 012001 (2013)

[21] G. Antchev et al. (TOTEM Collaboration), Nucl. Phys. B899, 527 (2015), 1503.08111

[22] R.U. Abbasi et al. (Telescope Array), Phys. Rev. D92, 032007 (2015), 1505.01860

[23] F. Abe et al. (CDF Collaboration), Phys. Rev. D 50, 5550 (1994)

[24] G.J. Alner et al. (UA5 Collaboration), Z. Phys. C32, 153 (1986)

[25] C. Avila et al. (E811 Collaboration), Phys. Lett. B445, 419 (1999)

[26] R.M. Baltrusaitis, G.L. Cassiday, J.W. Elbert, P.R. Gerhardy, S. Ko, E.C. Loh, Y. Mizumoto, P. Sokolsky, D. Steck, Phys. Rev. Lett. 52, 1380 (1984)

[27] M. Bozzo et al. (UA4 Collaboration), Phys. Lett. B147, 392 (1984)

[28] C. Patrignani et al. (Particle Data Group), Chin. Phys. C40, 100001 (2016)

[29] D.A. Fagundes, M.J. Menon, P.V.R.G. Silva, Journal of Physics G: Nuclear and Particle Physics 40, 065005 (2013)

[30] C. Bourrely, J.M. Myers, J. Soffer, T.T. Wu, Phys. Rev. D 85, 096009 (2012)

[31] Petrov, V.A., Prokudin, A.V., Eur. Phys. J. C 23, 135 (2002)

[32] M.M. Block, F. Halzen, Phys. Rev. D83, 077901 (2011), 1102.3163

[33] M.M. Islam, R.J. Luddy, A.V. Prokudin, Modern Physics Letters A 18, 743 (2003)

[34] L.L. Jenkovszky, A.I. Lengyel, D.I. Lontkovskyi, Int. J. Mod. Phys. A26, 4755 (2011), 1105. 1202

[35] M.M. Block, Phys. Rev. D 84, 091501 (2011)

[36] G. Antchev et al. (TOTEM) (2016), 1610.00603

[37] M. Aaboud, G. Aad, B. Abbott, J. Abdallah, O. Abdinov, B. Abeloos, R. Aben, O. AbouZeid, N. Abraham, H. Abramowicz et al. (ATLAS), Physics Letters B 761, 158 (2016) 